\title{
Das Jahrbuch wird 25
}

Das Jahrbuch erscheint jetzt im 25. Jahr. In dem vergangenen Vierteljahrhundert hat sich viel geändert. Der systematischen Anstöße wie in jenen Jahren ihrer Anfänge bedarf die Exilforschung nicht mehr. Die euphorischen Zeiten sind längst vorbei, in denen bis dahin unbekanntes Neuland großflächig erschlossen und eine weitere Öffentlichkeit darüber aufgeklärt werden konnte, welchen unwiederbringlichen Verlust die Vertreibung der vom "anderen Deutschland" repräsentierten einzigartigen Kultur nach 1933 bedeutet. Begleitet worden war dieser Forschungsprozess von einer Überprüfung herkömmlicher theoretischer und methodischer Vorgehensweisen; der Gegenstand hatte die Fragestellungen politisiert und zu transdisziplinären Forschungsperspektiven gezwungen. Aus der Rückschau sind jene Aufbrüche erfolgreich gewesen, der Preis aber ist, dass die Exilforschung zu einer normalen Wissenschaft geworden ist, wenngleich ihre nach wie vor nicht zu übersehende Außenseiterexistenz sie vor den Abgründen des allzu banalen Mainstream immunisierte.

Dennoch hat sich das Jahrbuch nicht überflüssig gemacht. Immer noch gilt es, weiße Flecken in der Forschungslandschaft aufzudecken. Auch das diesjährige Rahmenthema macht dies deutlich. Es beleuchtet ein bisher weitgehend missachtetes Forschungsfeld, wobei die einzelnen Beiträge auf unterschiedliche Weise erkennen lassen, wie sich inzwischen die theoretischen Grundlagen und methodischen Zugriffe verändert haben. Das Interesse wird sich künftig aber ebenso den Anschlussmöglichkeiten zu zentralen Themenbereichen der Geschichts-, Literatur- und Sozialwissenschaften, der Alltags-, Frauen- und Genderforschung sowie der Kultur- und Kommunikationswissenschaften zuwenden. Hingewiesen sei ferner auf die Grenzüberschreitungen zu anderen Exilen, die in komparativer Absicht, stärker als bisher geschehen, in den Blick zu nehmen sein werden. Die thematische Öffnung und zugleich methodische Rekonstruktion des deutschen Exils nach 1933, um die sich Herausgeber sowie Autorinnen und Autoren seit jeher bemüht haben, sind Ausdruck einer zunehmend historisierenden Wahrnehmung der Exile des 20. Jahrhunderts sowie ihrer Identifizierung und Deutung als Sonderformen der Moderne.

Der 25. Band lädt ein zur Rechenschaft über das bisher Geleistete. Davon soll die im Anhang abgedruckte Bibliografie einen kleinen Eindruck geben. Erst in der thematischen Ordnung ihres zweiten Teils wird deutlich, welches breite Feld der Forschungsgegenstände die insgesamt fast 380 seit 1983 erschienenen Artikel widerspiegeln. Über deren Rezeption kann nicht geklagt 
VIII Das Jahrbuch wird 25

werden; das Jahrbuch gehört seit Langem zum festen Kanon in den Zitationen von Veröffentlichungen zur Exilforschung. Als äußeren, symbolischen Anstoß, auch in der Zukunft auf diesem Pfad erfolgreich weiterzugehen, haben sich die Redaktion und der Verlag auf ein neues Erscheinungsbild verständigt, das künftigen Beiträgerinnen und Beiträgern zusätzlich motivierende Impulse in einem hoffentlich weiterhin dynamischen Forschungsprozess geben möge. 